\title{
PLATELET INDICES AS USEFUL INDICATORS OF NEONATAL SEPSIS
}

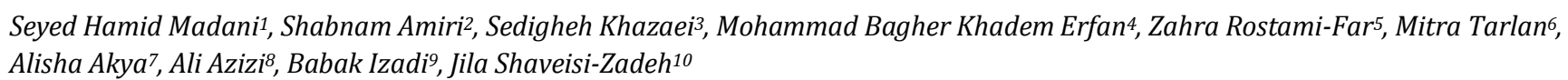

1 Molecular Pathology Research Center, Imam Reza Hospital, Kermanshah University of Medical Sciences, Kermanshah, Iran. 2Molecular Pathology Research Center, Imam Reza Hospital, Kermanshah University of Medical Sciences, Kermanshah, Iran. 3Molecular Pathology Research Center, Imam Reza Hospital, Kermanshah University of Medical Sciences, Kermanshah, Iran. ${ }^{4}$ Department of Molecular Medicine, Faculty of Medicine, Kurdistan University of Medical Sciences, Sanandaj, Iran. ${ }_{5}^{5}$ Molecular Pathology Research Center, Imam Reza Hospital, Kermanshah University of Medical Sciences, Kermanshah, Iran. ${ }^{6}$ Molecular Pathology Research Center, Imam Reza Hospital, Kermanshah University of Medical Sciences, Kermanshah, Iran. ${ }^{7}$ Nosocomial Infection Research Center, Faculty of Medicine, Kermanshah University of Medical Sciences, Kermanshah, Iran. ${ }^{8}$ Department of Community Medicine, Faculty of Medicine, Kermanshah University of Medical Sciences, Kermanshah, Iran. ${ }^{9}$ Molecular Pathology Research Center, Imam Reza Hospital, Kermanshah University of Medical Sciences, Kermanshah, Iran. ${ }_{10}$ Student Research Committee, Kermanshah University of Medical Sciences, Kermanshah Iran.

\section{BACKGROUND}

ABSTRACT

Neonatal sepsis is a disease process, which represents the consequences of systemic response to bacteria entering the blood stream during the first 28 days of life. Platelet count (PLT) and indices, including mean platelet volume (MPV), platelet distribution width (PDW) and Platelet larger cell ratio (P-LCR) are considered as markers of production rate and platelet activation. They are readily available via blood tests. However, their prognostic value in neonatal sepsis has not been fully clarified and several studies reported contradictory results. This study evaluated the changes of platelet count and indices in neonatal sepsis.

\section{METHODS}

This study was performed on 20 neonates with culture proven sepsis and 20 neonates with the similar age and weight but without any infectious disease served as the control group from February 2013 to June 2017 in the west of Iran. PLT, MPV, PDW, P-LCR and other infection markers (white blood cell count [WBC], erythrocyte sedimentation rate [ESR], and neutrophil percentage were also determined. This data was compared between patients and control group.

\section{RESULTS}

In sepsis group, MPV, PDW and P-LCR were significantly increased ( $\mathrm{p}=0.024, \mathrm{p}=0.006$ and $\mathrm{p}=0.005$ respectively), but WBC, Neutrophil percentage and PLT were not significantly different compared to controls. There was no significant difference in the platelet count and indices between Gram-negative and Gram-positive sepsis.

\section{CONCLUSIONS}

Diagnostic value of platelet indices is higher than that of WBC and neutrophil percentage. Therefore, platelet indices as inexpensive and easily available tests can be routinely performed for all neonates suspected to be in sepsis.

HOW TO CITE THIS ARTICLE: Madani SH, Amiri S, Khazaei S, et al. Platelet indices as useful indicators of neonatal sepsis. J. Evolution Med. Dent. Sci. 2019;8(20):1612-1617, DOI: 10.14260/jemds/2019/357

\section{BACKGROUND}

Neonatal sepsis is a disease process, which represents the systemic response of bacteria entering the bloodstream during the first 28 days of life. According to the National Neonatal Perinatal Database 2002-2003, the incidence of neonatal sepsis is 30 per live birth.(1) It is a very serious condition and if not diagnosed and treated quickly, can lead to shock, multiple organ dysfunction, permanent disability or death.(2) According to the onset of age, neonatal sepsis is divided into early-onset sepsis ( $\leq 3$ days of birth) and lateonset sepsis (After 3 days). The early-onset sepsis usually results from organisms acquired intrapartum, but late-onset sepsis is usually acquired from the environment.(3)

'Financial or Other Competing Interest': None.

Submission 21-02-2019, Peer Review 01-05-2019,

Acceptance 07-05-2019, Published 20-05-2019.

Corresponding Author:

Zahra Rostami-Far,

Molecular Pathology Research Center,

Imam Reza Hospital,

Zakaria Razi Bol, Kermanshah Iran.

E-mail: zahrarastamifar67@gmail.com

DOI: $10.14260 /$ jemds $/ 2019 / 357$
Neonatal sepsis occurs in one to four cases per 1000 live births in the developed countries, while it has been 10 times greater in many developing countries.(4) Group B Streptococcus, Escherichia coli, coagulase-negative Staphylococcus, Staphylococcus aureus, Klebsiella, Pseudomonas and Enterobacter are the most common cause of neonatal sepsis.(5) Diagnosis of neonatal sepsis remains one of the challenges that paediatrics are facing, because clinical findings are nonspecific and vague.(6) Almost all body systems and organs include haemostatic system can affected by sepsis. In this situation, clotting cascades do not function, many inflammatory cytokines released from endothelial and mononuclear cells, thrombosis take place at later stages and stimulation of plasminogen and activation of antithrombin occurs in fibrinolytic system.(7) As a result, fibrinogen and fibrinolytic substances are exhausted, and at the same time clots formation and bleeding associated with disseminated intravascular coagulation (DIC) occur. (8) DIC are associated with increased platelet destruction which cause thrombocytopenia. Thrombocytopenia is usually seen even before the bacteria are cultured from the blood.(9) Moreover, bacteria or its products may cause damage to endothelial leading to platelet adhesion and aggregation or may bind 
directly to platelets leading to aggregation and expedited elimination from blood circulation.(10) The normal ranges of platelet counts in neonates are similar to adults and are 150.000-400.000/ $\mu \mathrm{L}$. Platelet counts in approximately $40 \%$ of patients with severe sepsis are less than $80.000 / \mu \mathrm{L}$. ${ }^{(2)}$ Mean platelet volume (MPV) refers to average size of platelets and considered as markers of production rate and platelet activation. Normal ranges of MPV are between 8.5fl and 12.5fl.(11) The MPV levels increase in destructive thrombocytopenia and decrease in hypo proliferative thrombocytopenia.(12) Platelet distribution width (PDW) is an indicator of variation in platelet size. Normal ranges of PDW are between $10 \%$ and $17 \%$. The PDW levels increases in platelet consumption when turnover is increased, and behaviour similar to MPV during acute severe infections.(13) Platelet-large cell ratio (P-LCR) indicates the proportion of platelets greater than $12 \mathrm{fL}$ and the normal range is below 30 percent in the total platelet count. These indices can be measured by an inexpensive and readily available routine blood count.(14)

The usage and application of MPV, PDW and P-LCR in neonatal sepsis remains unknown and there are conflicting data about how sepsis affects these indices.(15,16) So the aim of this study was to investigate the changes of platelet count, MPV, PDW and P-LCR in neonatal sepsis.

\section{METHODS}

\section{Patients and Study Design}

This prospective case-control study was conducted between February 2013 and June 2017 in the West of Iran. Using convenient sampling method, 20 neonates with sepsis and 20 normal neonates without any medical problems, as the control group were selected. Protocol of the study was approved by Institute's ethical committee with informed and written consent obtained from the parents of all enrolled neonates.

This study was aimed to investigate the relation between neonatal sepsis and platelets and its indices (Platelet count, MPV, PDW). Neonates admitted in the neonatal intensive care unit of hospital and displaying signs and symptoms of sepsis, and/or born to mothers with risk factor for sepsis were included in this study. The inclusion criteria consisted of all neonates $(<28$ days) presenting with clinical signs and symptoms such as reduced activity, lethargy, hypotonia, hyporeflexia, apnoea, cyanosis, respiratory distress, irritability, hypothermia (temperature below $35 \mathrm{C}$ ), hyperthermia (Temperature above $40^{\circ} \mathrm{C}$ ), diarrhoea, vomiting and poor breast feeding and laboratory findings such as leukopenia or leukocytosis, increase of neutrophil percentage and erythrocyte sedimentation rate (ESR).(5)

Weight and gestational age matched healthy neonates without any infectious disease served as control. The groups were compared for age, gender, WBC count, neutrophil count, platelet count, PDW and MPV. Thrombocytopenia was divided into three categories: mild $\left(100,000-150,000 / \mathrm{mm}^{3}\right)$; moderate $\left(50,000-100,000 / \mathrm{mm}^{3}\right)$; and severe (s $50,000 / \mathrm{mm}^{3}$ ). Neutropenia was divided into three categories: mild $\left(1,000-1,500 / \mathrm{mm}^{3}\right)$; moderate $\left(500-1,000 / \mathrm{mm}^{3}\right)$ and severe $\left(\leq 500 / \mathrm{mm}^{3}\right)$.
The sensitivity, specificity, positive predictive value (PPV), and negative predictive value (NPV) of these tests were calculated.

The cut-off point kept for MPV in our study was 7.6 FL. The reference values were $4-10 \times 1000 / \mathrm{mm}^{3}$ for $\mathrm{WBC}, 40 \%-$ $70 \%$ for neutrophil, $140-440 \times 1000 / \mathrm{mm}^{3}$ f or PLT, $8.5-12.5$ FL for the MPV, 10-17 fl for the PDW and 17-45 \%for P-LCR

Blood culture is a gold standard for the diagnosis of sepsis, but since the positive result of blood culture may be false due to contamination, according to paediatric examination, only neonates have clinical and laboratory symptoms and their blood culture results were positive is considered as a real sepsis. ${ }^{(4)}$

\section{Bacterial Identification}

$1 \mathrm{ml}$ blood samples were drawn aseptically from all neonates with suspected sepsis, inoculated into a heart infusion broth, transported to respective laboratory and incubated at $37^{\circ} \mathrm{C}$, then was daily checked for signs of bacterial growth up to 7 days. For a positive broth culture, sub-cultures were done on MacConkey agar, blood agar and chocolate agar then incubated at $37^{\circ} \mathrm{C}$ for $24 \mathrm{~h}$. Blood culture broths with no bacterial growth after 7 days were sub-cultured and if no growth occurred, were reported as a negative. Bacterial isolates were identified by colony morphology, gram staining and standard biochemical tests.(9)

\section{Complete Blood Count}

$1 \mathrm{ml}$ blood samples were taken from all the patients and controls and collected in tubes containing EDTA. The complete blood counts (CBCs) performed using a Sysmex XT1800i analyser (Germany). Manufacturer's original kits were used in analyses. The normal range of PLT, MPV and PDW were $150,000-400,000 / \mu \mathrm{L}, \quad 8.5-12.5$ fl and $10-17 \%$ respectively.

\section{Sample Size Calculation}

Calculation of sample size was done by using simplified formula for difference in means of power $90 \%$ and confidence interval $95 \%$ and substituting values of parameters of MPV (mean, SD ) of sepsis patients and controls obtained by study done by Choudhary et al. (17) as shown below:

Where $\mathrm{n}=$ total sample size $\quad n_{1}=n_{2}=\frac{\left(S_{1}{ }^{2}+S_{2}{ }^{2}\right)\left(Z_{1-\frac{\alpha}{2}}+Z_{1-\beta}\right)^{2}}{\left(\bar{X}_{1}-\bar{X}_{2}\right)^{2}}$

$\overline{\mathrm{X}}=$ mean

$\mathrm{S}=$ standard deviation

$\mathrm{Z}_{(1-(\alpha / 2))}=$ related to the chosen significance criterion $\alpha$; can be found in normal distribution tables. For confidence interval of $95 \%, \alpha=0.05$ (for confidence interval of $95 \%$ )

$\mathrm{Z}(1-\beta)=$ related to the chosen power can be found in normal distribution tables. For power of $90 \%, \beta=0.1$ (for power of power $90 \%$ )

$\overline{\mathrm{X}}_{1}=11.82$

$\overline{\mathrm{X}}_{2}=9.75$

$S_{2}^{2}=(1.45)^{2}$

$$
S_{1}^{2}=(1.69)^{2}
$$

$\mathrm{Z}_{1-\beta}=1.29$ 


\begin{tabular}{|c|c|c|c|}
\hline Rows & Bacteria & Number & Percent \\
\hline 1 & Escherichia coli & 2 & $10 \%$ \\
\hline 2 & Non-haemolytic streptococcus & 1 & $5 \%$ \\
\hline 3 & Staphylococcus coagulase negative & 1 & $5 \%$ \\
\hline 4 & Beta haemolytic streptococcus & 2 & $10 \%$ \\
\hline 5 & Citrobacter & 8 & $40 \%$ \\
\hline 6 & Enterobacter & 1 & $5 \%$ \\
\hline 7 & Staphylococcus aureus & 2 & $10 \%$ \\
\hline 8 & Pseudomonas & 1 & $5 \%$ \\
\hline \multirow[t]{2}{*}{9} & Enterococci & 2 & $10 \%$ \\
\hline & Total & 20 & $100 \%$ \\
\hline \multicolumn{4}{|c|}{$\begin{array}{c}\text { Table 1. Types and Frequency of Bacteria Isolated from } \\
\text { Blood Cultures }\end{array}$} \\
\hline
\end{tabular}

\begin{tabular}{|c|c|c|c|}
\hline $\begin{array}{c}\text { Type of } \\
\text { Microorganism }\end{array}$ & $\begin{array}{c}\text { No. (\%) of } \\
\text { Early Sepsis }\end{array}$ & $\begin{array}{c}\text { No. (\%) of Late } \\
\text { Sepsis }\end{array}$ & $\begin{array}{c}\text { p- } \\
\text { Value }\end{array}$ \\
\hline Escherichia coli & $1(\% 10)$ & $1(\% 10)$ & 1.000 \\
\hline $\begin{array}{c}\text { Non-haemolytic } \\
\text { streptococcus }\end{array}$ & $1(\% 10)$ & 0 & 0.3 \\
\hline $\begin{array}{c}\text { Beta haemolytic } \\
\text { streptococcus }\end{array}$ & $1(\% 10)$ & $1(\% 10)$ & 1.000 \\
\hline Citrobacter & $4(\% 40)$ & $4(\% 40)$ & 1.000 \\
\hline $\begin{array}{c}\text { Staphylococcus } \\
\text { aureus }\end{array}$ & $2(\% 20)$ & 0 & 0.136 \\
\hline Pseudomonas & $1(\% 10)$ & 0 & 0.3 \\
\hline $\begin{array}{c}\text { Staphylococcus } \\
\text { coagulase negative }\end{array}$ & 0 & $1(\% 10)$ & 0.3 \\
\hline Enterobacter & 0 & $1(\% 10)$ & 03 \\
\hline Enterococci & 0 & $2(\% 20)$ & \\
\hline Total & $10(\% 100)$ & $10(\% 100)$ & 0.136 \\
\hline
\end{tabular}

Table 2. Frequency of Bacterial Isolates in Neonates with Early and Late Sepsis

\begin{tabular}{|c|c|c|c|}
\hline & Sepsis Group & $\begin{array}{l}\text { Control } \\
\text { Group }\end{array}$ & p Value \\
\hline Age (days) & 7.5 & 2,6 & $\mathrm{p}=0.042$ \\
\hline Sex (M:F) & $10: 10$ & $12: 8$ & \\
\hline \multicolumn{4}{|l|}{$\begin{array}{l}\text { Laboratory findings } \\
\qquad(\text { Mean } \pm \text { SE) }\end{array}$} \\
\hline $\mathrm{WBC}(/ \mu \mathrm{L})$ & $14.84 \pm 2.94$ & $14.79 \pm 2,98$ & $\mathrm{p}=0.99$ \\
\hline Neutrophil (\%) & $61.89 \pm 14.34$ & $52,77 \pm 3,92$ & $\mathrm{p}=0,54$ \\
\hline \multicolumn{4}{|c|}{$\begin{array}{l}\text { Table 3. Demographic Characteristics and Laboratory } \\
\text { Findings of Patients and Control Group }\end{array}$} \\
\hline $\begin{array}{l}\text { Age, sex and labc } \\
\text { and controls are sho } \\
\text { was significantly hig } \\
\text { differences were }\end{array}$ & ratory finding of & $\begin{array}{l}\text { f two groups } \\
\text { In this study, }\end{array}$ & $\begin{array}{l}\text { of patients } \\
\text { mean age }\end{array}$ \\
\hline
\end{tabular}

\begin{tabular}{|c|c|c|c|c|}
\hline & $\begin{array}{c}\text { Minimum- } \\
\text { Maximum }\end{array}$ & $\begin{array}{c}\text { Sepsis Group } \\
\text { (Mean } \pm \text { SE) }\end{array}$ & $\begin{array}{c}\text { Control } \\
\text { Group } \\
\text { (Mean } \pm \text { SE) }\end{array}$ & $\begin{array}{c}\mathbf{p} \\
\text { Value }\end{array}$ \\
\hline \multirow{2}{*}{ PLT } & $140-400 / \mu \mathrm{L}$ & $\begin{array}{c}243.4 \pm \\
28.31\end{array}$ & $\begin{array}{c}237,65 \pm \\
21,74\end{array}$ & 0.87 \\
\hline \multirow{2}{*}{$\mathrm{MPV}$} & \multirow{2}{*}{$8.5-12.5 \mathrm{fl}$} & $\begin{array}{c}10.3 \pm \\
0.23\end{array}$ & $\begin{array}{c}9.3 \pm \\
0.19\end{array}$ & 0.005 \\
\hline \multirow{2}{*}{ PDW } & $10-17 \%$ & $\begin{array}{c}13.5 \pm \\
0.5\end{array}$ & $\begin{array}{c}11.7 \pm \\
0.27\end{array}$ & 0.005 \\
\hline \multirow{2}{*}{ P-LCR } & $17-45 \%$ & $\begin{array}{c}28.28 \pm \\
1.44\end{array}$ & $\begin{array}{c}21,59 \pm \\
1,16\end{array}$ & 0.001 \\
\hline
\end{tabular}

Table 4. Comparison of Platelet Count and Their Indices between Sepsis Patients and Controls

In sepsis group, MPV, PDW and P-LCR was significantly increased compared to the control group.

\begin{tabular}{|c|c|c|c|}
\hline & \multicolumn{2}{|c|}{ Gram Stain } & p value \\
\hline & $\begin{array}{c}\text { Negative (Mean } \pm \\
\text { SE) }\end{array}$ & Positive (Mean \pm SE) & \\
\hline PLT & $228.72 \pm 31.30$ & $261.88 \pm 51.54$ & $\mathrm{p}=0.56$ \\
\hline MPV & $10.8 \pm 0.31$ & $10.56 \pm 0.35$ & $\mathrm{p}=0.31$ \\
\hline PDW & $13.3 \pm 0.63$ & $13.6 \pm 0.87$ & $\mathrm{p}=0.79$ \\
\hline P-LCR & $27.4 \pm 1.86$ & $29.3 \pm 2.35$ & $\mathrm{p}=0.53$ \\
\hline
\end{tabular}

Table 5. Comparison of Platelet Count and Indices in Sepsis Patients According to Gram Staining

Value of PLT count, PDW, MPV and P-LCR among Gramnegative and Gram-positive infected patients. There was no significant differences between two group. There were no significant differences for platelet indices in neonates with early and late sepsis.

\begin{tabular}{|c|c|c|c|}
\hline & \multicolumn{2}{|c|}{ Stage } & p Value \\
\hline & $\begin{array}{c}\text { Early onset sepsis } \\
(\text { Mean } \pm \text { SE) }\end{array}$ & $\begin{array}{c}\text { Late onset sepsis } \\
(\text { Mean } \pm \text { SE) }\end{array}$ & \\
\hline PLT & $238,6 \pm 24,31$ & $248,2 \pm 52,80$ & $\mathrm{p}=0.87$ \\
\hline MPV & $10.19 \pm 0.33$ & $10.41 \pm 0.33$ & $\mathrm{p}=0.65$ \\
\hline PDW & $13.17 \pm 0.79$ & $13.84 \pm 0.66$ & $\mathrm{p}=0.52$ \\
\hline P-LCR & $27.54 \pm 2,20$ & $29.02 \pm 1.97$ & $\mathrm{p}=0.62$ \\
\hline
\end{tabular}

Table 6. Comparison of Platelet Count and Their Indices in Neonates with Early and Late Sepsis

\begin{tabular}{|c|c|c|c|c|c|c|c|c|}
\hline Parameter & AUC & Cut-off & $\begin{array}{c}\text { Sensitivity } \\
\text { (\%) }\end{array}$ & $\begin{array}{c}\text { Specificity } \\
(\mathbf{\%})\end{array}$ & $\begin{array}{c}\text { PPV } \\
\text { (\%) }\end{array}$ & $\begin{array}{c}\text { NPV } \\
(\mathbf{\%})\end{array}$ & LR+ & $\begin{array}{c}\text { LR } \\
-\end{array}$ \\
\hline MPV & 0.73 & 9.95 & 65.3 & 75 & 31.81 & 27.78 & 2.6 & 0.47 \\
\hline PDW & 0.74 & 12.35 & 70 & 70.5 & 30 & 30 & 2.37 & 0.42 \\
\hline PLCR & 0.77 & 25.20 & 65.2 & 80 & 35 & 23.53 & 3.26 & 0.43 \\
\hline
\end{tabular}

Table 7. Diagnostic Value Analysis for Significant Different Data Between Sepsis Groups and Control Group

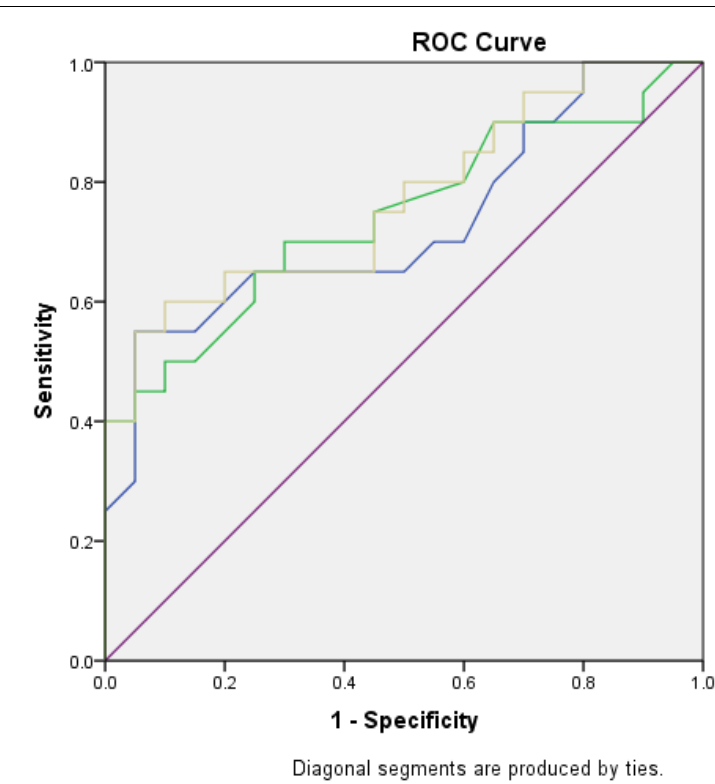

Figure 1. Receiver Operating Characteristic Curve (ROC) for The Predictors of Cases with Sepsis

Area under Curve (AUC)- MPV (0.73), RDW (0.74), PLCR (0.77), Cutoff Value- MPV (9.95), RDW (12.35), PLCR (25.20), Sensitivity and Specificity- MPV (65.3 and 75), RDW (70 and 70.5), PLCR (65.2 and 80) (Figure 1, Table 7).

We obtained sample size of 13 in each group. To enhance the power of study, we increased the number of cases to 20 in case and control group. 


\section{Data Analysis}

Data were collected and entered into the Statistical Package for Social Science (IBM SPSS) version 16 software. All data were analysed in terms of mean + standard deviation (SD). The comparison between groups regarding qualitative data was analysed by using the Chi-square test, while comparisons of parametric data with parametric distribution between the study subjects and controls were performed by using independent t-test.

In addition, the sensitivity, specificity, cut-off points, positive and negative predictive values and likelihood ratios were calculated by the area under the ROC curve. The confidence interval (CI) was set at $95 \%$ and $\mathrm{P}$ value $<0.05$ was considered statistically significant.

\section{RESULTS}

This survey was performed in the neonatal intensive care unit (NICU) of Imam Reza Hospital of Kermanshah (750 beds, 29 sections, Governmental, general high direct referral rate admission) in the west of Iran. From 20 newborns with sepsis, 10 were males $(50 \%)$ and 10 were females (50\%). The frequency of mild, moderate and sever thrombocytopenia was $15 \%, 5 \%$ and $5 \%$ respectively.

Nine species of bacteria were isolated during the study, the number and frequency of each species been shown in Table 1. The frequency of isolated gram-negative and gram-positive bacteria was $55 \%$ and $45 \%$, respectively. The most frequently isolated bacteria were Citrobacter species. Table 2 shows Citrobacter is the most common organism in both early and late sepsis.

\section{DISCUSSION}

The relationship between platelet indices and various diseases has been the subject of increasing study because of their rapid and widespread availability. Haematology analysers with coulter principle, measure platelet volume by changes of electrical properties, which depends on platelet vertical diameter; other analysers based on laser optical technology determine platelet volume by cross diameter of platelet. Regardless of the method of measurement, activated platelets seem larger. MPV is inversely commensurate to the rate of maturation of platelet.(5) MPV value decreased in conditions, which are associated with reduction of platelet production in the bone marrow, such as aplastic anaemia and myelodysplastic syndrome and chemotherapy induced bone marrow suppression.(11) Increases of MPV value suggests that proportion of younger platelets in the blood circulation are increased, and is indicate increased platelet destruction or production, such as hypersplenism and immune thrombocytopenia.(13) PDW is an indicator of the heterogeneity in platelet size. Increases of PDW suggest a large range of platelet because of swelling, immaturity and destruction.(12)

Thrombocytopenia is a common hematologic finding in critically ill patients, and is associated with increased mortality.(18-20) It is also a known fact in preterm infants with sepsis. A close relationship between sepsis and thrombocytopenia has been suggested in some studies. Mannan et al. in their study showed that 50\% cases of neonatal sepsis had thrombocytopenia.(21) Another study by Guida et al. showed that $54 \%$ of very low birth weight neonates with culture proven neonatal sepsis, developed thrombocytopenia.(22) Abdulla et al showed that $42.8 \%$ babies with sepsis had thrombocytopenia.(23) Thrombocytopenia was found in $25 \%$ of our neonatal population, which is lower than previous studies.

Neonatal sepsis refers to generalized bacterial infection and is a serious condition requires rapid and accurate diagnosis. There have been a few reports which have evaluated the relationship between platelet indices and sepsis disorder.(15) In the present study, we found significant increased levels of MPV, PDW and P-LCR in neonates with sepsis than healthy neonates, In addition, a lower platelet count was observed in septic neonates, but it is not statistically significant. Therefore, platelet indices may be considered as early predictor for diagnosis of neonatal sepsis.

Clinical studies that have examined the association between platelet count and indices and sepsis demonstrate contradictory results. In a study conducted in newborns, Patrick et al reported that levels of MPV and PDW were higher in patients with bacteraemia than controls. (24) Guclu et al reported significant change in MPV in patients with sepsis compared to healthy controls, but platelet count was not significantly different.(16) Van der Lelie et al showed that MPV was increased in 13 of the 25 sepsis patients, and returned to normal values after the disease was controlled.(25) In a cohort study performed on newborn with sepsis, thrombocytopenia and elevated MPV were prominent features.(26) Conversely, Acikgoz et al. reported that MPV and PDW not significantly different between sepsis patients with DIC and without DIC.(27) In addition to sepsis, MPV and PDW also have a positive correlation with many different diseases such as diabetes, myocardial infarction and various infections. (28) Elevated white blood cell and neutrophil counts are the main laboratory findings in the diagnosis of sepsis, but these factors were not statistically significant in our study.(5) These findings suggested that platelet count and platelet indices may serve as useful indicators of neonatal sepsis. However, no significant differences were found in either platelet count or MPV and PDW of neonates between those with early and late on-set sepsis.

It was observed in this study that Gram-negative bacteria are more commonly isolated in compared to Gram-positive in neonates with sepsis which is similar to the results of the majority of previous studies.(29) Decrease of platelet count was more in Gram-negative infections as compared to grampositive infection. Also, MPV was increased in in Gramnegative infections as compared to Gram-positive infection but not statistically significant. In a study, Yilmaz et al found that PDW was increased in the presence of endotoxin in animals with Gram-negative sepsis.(30) Aydemir et al reported that MPV was significantly increased for the first 3 days of patients with Gram-positive sepsis, for 4 days in Gramnegative septic patients, and for all 5 days in fungal septic patients. (31)

The exact mechanisms of increases of MPV and PDW in neonatal sepsis are not entirely clear. It has been reported that coagulation and platelet activation can occur during early stages of sepsis. During activation process, platelets change in shape of the platelets from a discoid form to a spherical form with pseudopodia in order to acquire a larger surface, formation of pseudopodia can increase PDW value.(16,32) Also, some researches reports that MPV is associated with function and activation of platelet. Generally, increase rate of platelet consumption leads to the release of 
young platelets from the bone marrow. Young platelets are more active than older platelets and also have a larger size, so MPV is frequently elevated. Catal et al hypothesized that increased levels of $\beta$-thromboglobulin in bacterial infections might activate and release of platelets.(33) Another possible mechanism is related to the thrombopoietin (TPO). TPO is a liver-produced protein that regulates megakaryocytopoiesis and plays a role in the increase in platelet counts. Some studies reported a relationship between the TPO levels and MPV values. Some inflammatory cytokines such as interleukin 6 (IL-6) can induce production of TPO; since the IL-6, increased during sepsis, MPV might be increased due to increased TPO production induced by IL-6.(34)

Our findings suggested that MPV, PDW and PICR levels have acceptable sensitivity $(65.3 \%, 70 \%, 65.2 \%)$ and good specificity $(75 \%, 70 \%, 80 \%)$ for sepsis diagnosis. Therefore, platelet indices may be used as an additional test in the diagnosis of sepsis. This finding is higher than the study findings with that of Arad ID et al (1986) who showed that specificity of MPV in neonatal sepsis is $54 \%$ and $46 \%$ respectively.(35)

\section{Limitation}

The sample size of patients was not many, and our findings are not representative for all neonates with sepsis. Our laboratory used EDTA as an anticoagulant- and plateletindices might be affected by these anticoagulants. The best interval time for platelet indices measurement is within 2 hours after obtaining of blood samples, but it's may not be achieved.(5) Some antibiotics may have an impact on the platelet indices and could affect the results of our study, so future studies with larger sample size are needed to confirm our results.(26)

\section{CONCLUSIONS}

Neonates with sepsis have the significantly increased MPV, PDW and P-LCR compared to healthy neonates. Therefore, platelet indices, as part of a routine automated cell count, are cheap and readily available tests that can be used as valuable clues in the diagnosis of neonatal sepsis.

\section{ACKNOWLEDGEMENT}

The authors would like to thank the Clinical Research Development Center of Imam Reza Hospital for Consulting Services.

\section{REFERENCES}

[1] Report of the National Neonatal Perinatal Database (National Neonatology Forum) 2002-3.

[2] Grant $\mathrm{CH}$, Arnott A, Brook T, et al. Reducing antibiotic exposure in suspected neonatal sepsis. Clinical Pediatrics (Phila) 2018;57(1):76-81.

[3] Chiarot E, Spagnuolo A, Maccari S, et al. Protective effect of Group B Streptococcus type-III polysaccharide conjugates against maternal colonization, ascending infection and neonatal transmission in rodent models. Scientific Reports 2018;8(1):2593.

[4] Simonsen KA, Anderson-Berry AL, Delair SF, et al. Early-onset neonatal sepsis. Clinical Microbiology Reviews 2014;27(1):21-47.
[5] Rostami-Far Z, Ghadiri K, Rostami-Far M, et al. Glucose-6-phosphate dehydrogenase deficiency (G6PD) as a risk factor of male neonatal sepsis. Journal of Medicine and Life 2016;9(1):34-8.

[6] González-Vázquez P, Larrañeta E, McCrudden MTC, et al. Transdermal delivery of gentamicin using dissolving microneedle arrays for potential treatment of neonatal sepsis. Journal of Controlled Release 2017;265:30-40.

[7] Zardi E, Zardi D, Dobrina A, et al. Prostacyclin in sepsis: a systematic review. Prostaglandins \& Other Lipid Mediators 2007;83(1-2):1-24.

[8] Simmons J, Pittet JF. The coagulopathy of acute sepsis. Current Opinion in Anaesthesiology 2015;28(2):22736.

[9] Fitzgerald JR, Foster TJ, Cox D. The interaction of bacterial pathogens with platelets. Nature Reviews Microbiology 2006;4(6):445-57.

[10] Stevenson EK, Rubenstein AR, Radin GT, et al. Two decades of mortality trends among patients with severe sepsis: a comparative meta-analysis. Critical Care Medicine 2014;42(3):625-31.

[11] Gao Y, Li Y, Yu X, et al. The impact of various platelet indices as prognostic markers of septic shock. PLoS One 2014;9(8):e103761.

[12] Borkataky S, Jain R, Gupta R, et al. Role of platelet volume indices in the differential diagnosis of thrombocytopenia: a simple and inexpensive method. Hematology 2009;14(3):182-6.

[13] Ahmad MS, Waheed A. Platelet counts, MPV and PDW in culture proven and probable neonatal sepsis and association of platelet counts with mortality rate. J Coll Physicians Surg Pak 2014;24(5):340-4.

[14] Sachdev R, Tiwari AK, Goel S, et al. Establishing biological reference intervals for novel platelet parameters (immature platelet fraction, high immature platelet fraction, platelet distribution width, platelet large cell ratio, platelet-X, plateletcrit and platelet distribution width) and their correlations among each other. Indian Journal of Pathology and Microbiology 2014;57(2):231-5.

[15] Arif S, Ahmad I, Ali SM, et al. Thrombocytopenia and bacterial sepsis in neonates. Indian Journal of Hematology and Blood Transfusion 2012;28(3):14751.

[16] Guclu E, Durmaz Y, Karabay O. Effect of severe sepsis on platelet count and their indices. African Health Sciences 2013;13(2):333-8.

[17] Choudhary RR, Makwana M, Mourya HK, et al. Evaluation of platelet and its indices as a marker of neonatal sepsis: a prospective case control study. IJCP 2018;5(5):1898-903.

[18] Baughman RR, Lower EE, Flessa HC, et al. Thrombocytopenia in the intensive care unit. Chest 1993;104(4):1243-7.

[19] Hanes SD, Quarles DA, Boucher BA. Critical care: incidence and risk factors of thrombocytopenia in critically ill trauma patients. Annals of Pharmacotherapy 1997;31(3):285-9. 
[20] Stephan F, Montblanc J, Cheffi A, et al. Thrombocytopenia in critically ill surgical patients: a case-control study evaluating attributable mortality and transfusion requirements. Critical Care 1999;3(6):151-8.

[21] Mannan M, Shahidullah M, Noor MK, et al. Utility of Creactive protein and hematological parameters in the detection of neonatal sepsis. Mymensingh Medical Journal 2010;19(2):259-63.

[22] Guida JD, Kunig AM, Leef KH, et al. Platelet count and sepsis in very low birth weight neonates: is there an organism-specific response? Pediatrics 2003;111(6 Pt 1):1411-5.

[23] Alshorman A, Maghayreh M, Khriesat W, et al. The effect of neonatal sepsis on platelet count and their indices. Jordan Medical Journal 2008;42(2):82-6.

[24] Patrick CH, Lazarchick J. The effect of bacteremia on automated platelet measurements in neonates. American Journal of Clinical Pathology 1990;93(3):391-4.

[25] Van der Lelie J, Von dem Borne AK. Increased mean platelet volume in septicaemia. Journal of Clinical Pathology 1983;36(6):693-6.

[26] El-Mashad GM, El-Sayed HM, Rizk MS, et al. Mean platelet volume and serum uric acid in neonatal sepsis. Menoufia Medical Journal 2017;30(2):581-7.

[27] Acikgoz S, Akduman D, Eskici Z, et al. Thrombocyte and erythrocyte indices in sepsis and disseminated intravascular coagulation. Journal of Medical Biochemistry 2012;31(1):60-4.
[28] Lee IR, Shin JI, Park SJ, et al. Mean platelet volume in young children with urinary tract infection. Scientific Reports 2015;5:18072.

[29] Mouzinho A, Rosenfeld CR, Sánchez PJ, et al. Revised reference ranges for circulating neutrophils in verylow-birth-weight neonates. Pediatrics 1994;94(1):7682.

[30] Yilmaz Z, Eralp 0, Ilcol YO. Evaluation of platelet count and its association with plateletcrit, mean platelet volume, and platelet size distribution width in a canine model of endotoxemia. Veterinary Clinical Pathology 2008;37(2):159-63.

[31] Aydemir H, Piskin N, Akduman D, et al. Platelet and mean platelet volume kinetics in adult patients with sepsis. Platelets 2015;26(4):331-5.

[32] Vagdatli E, Gounari E, Lazaridou E, et al. Platelet distribution width: a simple, practical and specific marker of activation of coagulation. Hippokratia 2010;14(1):28-32.

[33] Catal F, Bavbek N, Bayrak O, et al. Platelet parameters in children with upper urinary tract infection: is there a specific response? Renal Failure 2008;30(4):377-81.

[34] Kaser A, Brandacher G, Steurer W, et al. Interleukin-6 stimulates thrombopoiesis through thrombopoietin: role in inflammatory thrombocytosis. Blood 2001;98(9):2720-5.

[35] Arad ID, Alpan G, Sznajderman SD, et al. The mean platelet volume (MPV) in the neonatal period. American Journal of Perinatology 1986;3(1):1-3. 